\title{
Dispersive OPA at Very Near-Infrared Wavelengths
}

\author{
Sarvagya Dwivedi ${ }^{1,}$ *, Mathias Prost ${ }^{1}$, Tangla David Kongnyuy ${ }^{1}$, Jon Ø. Kjellman ${ }^{1}$, Xavier Rottenberg ${ }^{1}$, \\ Roelof Jansen ${ }^{1}$, Wim Bogaerts ${ }^{2}$, Marcus S. Dahlem ${ }^{1}$ \\ ${ }^{I}$ Imec, Kapeldreef 75, 3001 Leuven, Belgium \\ ${ }^{2}$ Photonics Research Group, Ghent University-Imec, 9052 Ghent, Belgium \\ *Sarvagya.Dwivedi@imec.be
}

\begin{abstract}
We present a serpentine-based 16-element dispersive OPA at very near-IR wavelengths that leverages $\mathrm{SiN}$ waveguide feeding. 2D beam steering is achieved by wavelength sweep, with tunability of $0.11 \% \mathrm{~nm}$ on slow and $3.52 \% \mathrm{~nm}$ on fast axis.
\end{abstract}

Keywords: Integrated optics, Optical phased arrays, Silicon Nitride, LiDAR

\section{INTRODUCTION}

Integrated optical phased arrays (OPAs) have recently attracted much attention as they enable beam steering with low cost, size, weight, and power compared to bulk optics systems using moving parts [1]. They are ideal for various applications such as sensing, imaging, display technology and Light Detection and Ranging (LiDAR). Several integrated OPAs have been recently demonstrated for beam steering, either by wavelength dispersion or active phase control, or a combination of both [2-3]. In this work, we demonstrate a serpentine-based 16-element OPA [4] at very near-IR wavelengths that leverage Silicon Nitride ( $\mathrm{SiN}$ ) waveguide feeding. The 2D calibration-free [5] beam steering has been achieved in the dispersive OPA architecture by just a laser wavelength sweep [6]. This kind of architecture enables low phase errors, low power, compactness and scalability, and maximum usage of the emission area for future on-chip OPAs at these wavelengths.

\section{DESIGN AND FABRICATION}

The integrated dispersive OPA is fed with a SiN waveguide in a serpentine architecture, as shown in Fig. 1(a). The structure leverages low-loss bends [7] to keep the emission area as dense as possible. The bends are designed with a radius of $10 \mu \mathrm{m}$, as shown in Fig. 1(c). The antennas are implemented by using SiN perturbations on both sides of the waveguide, as shown in the inset of Fig. 1(d). These gratings are known as leaky wave antennas (LWAs), which have a weak strength that allows a large emission area. The strength can be well controlled by selecting the distance of the perturbations from the waveguide. The grating allows wavelength steering along the slow axis (angle $\theta_{y}$ ). The emission angle is given by $\sin \theta=\left(n_{e f f}-\lambda / P\right) / n_{c}$, where $n_{c}$ is the cladding index, $n_{e f f}$ the grating index, $P$ the period of the grating, and $\lambda$ the operating free-space wavelength. The fast axis (angle $\theta_{x}$ ) is steered through a constant delay length $(\Delta L)$ between the antennas. The phase delay is given by $\Delta \phi=2 \pi .\left(n_{e f f}(\lambda) . \Delta L\right) / \lambda$, which is a wavelength dependent expression with $n_{\text {eff }}(\lambda)$ as the effective index of the delay line.
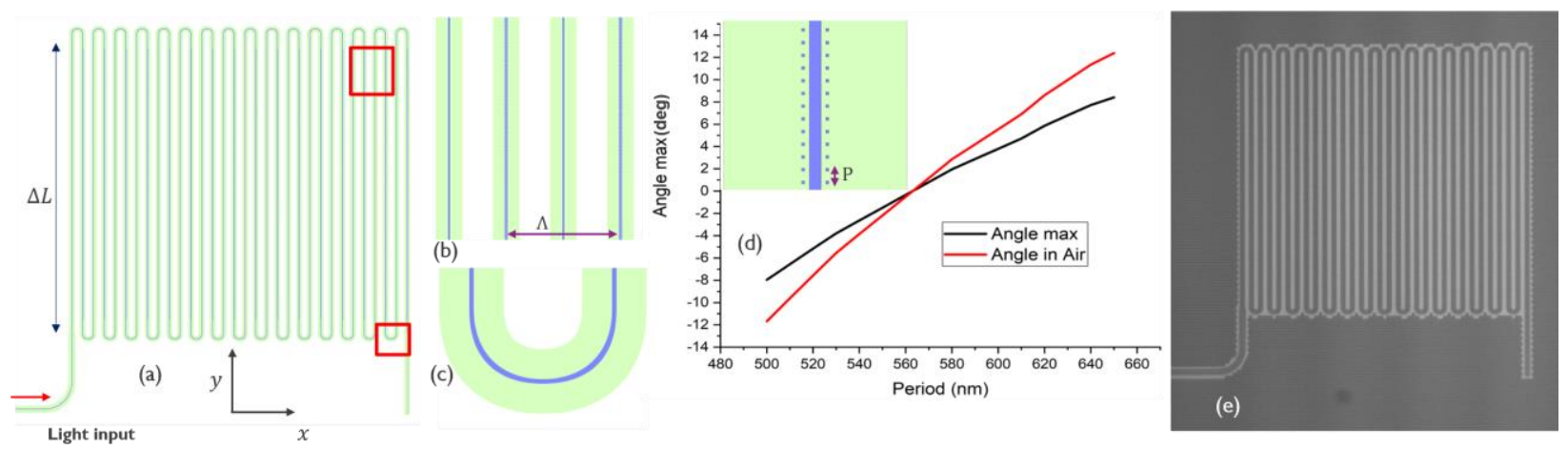

Fig. 1. (a) Designed OPA, $x$ is fast axis and $y$ is slow axis; (b) zoom-in image of alternate LWAs with a pitch $\Lambda$; (c) zoom-in image of a low-loss bend; (d) simulated emission angle in air for a period sweep, with a zoom-in of the LWA with grating period $P$; and (e) optical image of the fabricated OPA.

The rate at which the beam can be steered in the slow direction is given by $d \theta_{x} / d \lambda=d n_{\text {eff }} / d \lambda-1 / P$ (for small $\theta$, $\sin \theta \approx \theta$ ). The LWA is designed with a period $P=640 \mathrm{~nm}$ which corresponds to an emission angle of $10^{\circ}$ in air at a wavelength of $905 \mathrm{~nm}$, as shown Fig. 1(d). The LWA section is $500 \mu \mathrm{m}$ long, and the strength of the grating is aimed 
to a few $\mathrm{dB} / \mathrm{mm}$ in order to have most of the light emitted at the end of the OPA. The aperture size of the dispersive OPA is $0.5 \times 0.6 \mathrm{~mm}^{2}$. The OPA was fabricated on imec's BioPIX PECVD SiN platform in imec's $200 \mathrm{~mm}$ CMOS pilot line [8]. The stack consists of a $300 \mathrm{~nm}$-thick SiN device layer on $3.3 \mu \mathrm{m}$ of buried oxide on a Si wafer (the platform also has top heaters for active tuning of devices). Fig. 1(f) shows an optical image of the fabricated OPA.

\section{MEASUREMENT AND RESULTS}

The measurements were performed using a back-focal plane (BFP) imaging setup, where the far-field of the OPA is collected by a microscope objective and imaged on an infrared (IR) camera using a set of lenses. Laser light is coupled to the chip using SiN low-reflection vertical grating couplers. The measured SiN waveguide loss at $905 \mathrm{~nm}$ is 0.25 $\mathrm{dB} / \mathrm{cm}$. The captured far-field image plane of the OPA emission at a wavelength of $905 \mathrm{~nm}$ is shown in Fig. 2(a). For a given input wavelength, the OPA emits several beam spots. These are the grating lobes of the array which are related to the pitch $\Lambda$ of the LWAs. The resulting angular separation of the grating lobes is $1.35^{\circ}$. The figure inset shows a closer view of the captured beam-spot. Thanks to the SiN waveguide quality with low propagation phase errors, the beam-spot is well resolved and no significant sidelobes due to accumulated phase errors are observed. The beam divergence is $0.22^{\circ} \times 0.15^{\circ}$. As a $2 \mathrm{D}$ wavelength-based beam steering device, we investigated the tunability of the OPA. Fig. 2 (b) and (c) show the angular beam spot position as a function of the input wavelength for the fast and slow axis, respectively. We measured a tunability $d \theta_{y} / d \lambda=0.11 \% \mathrm{~nm}$ in the slow axis. This value is related to the design of the LWA. In the fast axis, we measured a tunability $d \theta_{x} / d \lambda=3.52 \% \mathrm{~nm}$, i.e. the unambiguous steering range is covered by a wavelength step of $0.38 \mathrm{~nm}$. Some points in the plot may appear digitized due to the resolution of the camera pixel. To illustrate the scanning properties of the OPA, we created a composite image of the points obtained from a wavelength scan. The result is show in Fig. 2(d) and covers a wavelength tuning from 895 to $920 \mathrm{~nm}$, with a $1 \mathrm{~nm}$ step for each acquisition.
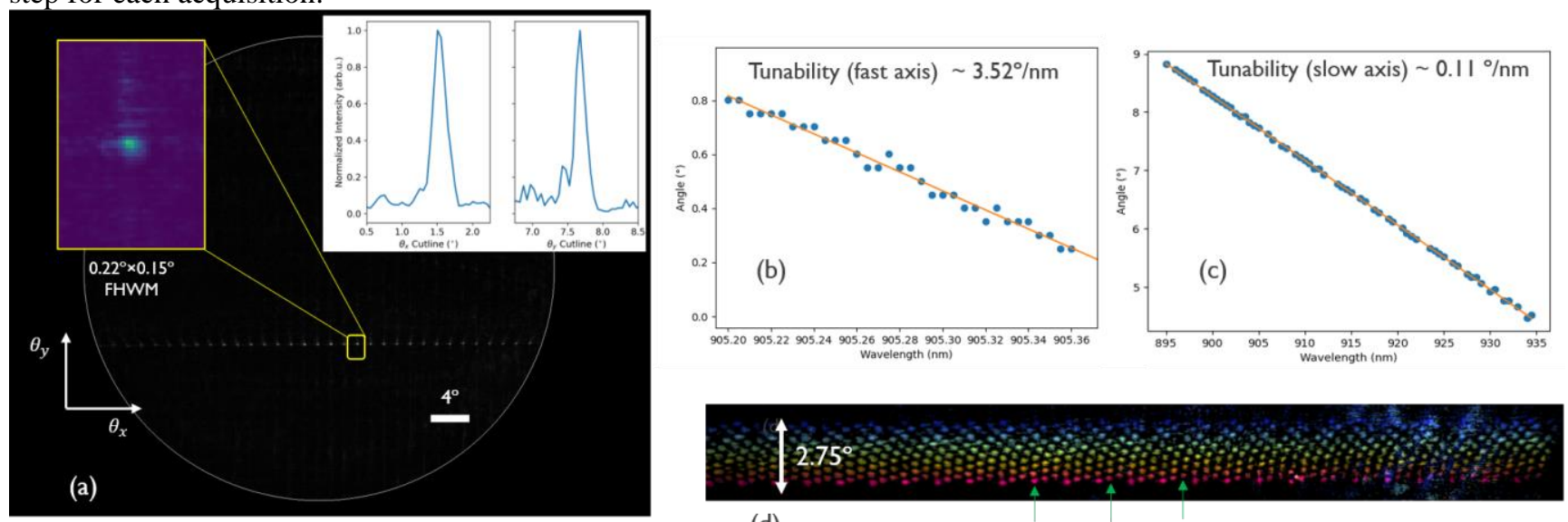

(d)

$895 \mathrm{~nm} 896 \mathrm{~nm} 897 \mathrm{~nm}$

Fig. 2. (a) Far-field of the measured dispersive OPA showing the beam spot and divergence; (b) measured OPA tunability in the fast axis; (c) measured OPA tunability in the slow axis; and (d) composite 2D beam steering image showing the point cloud in both directions, when the laser is swept from $895 \mathrm{~nm}$ to $920 \mathrm{~nm}$ with a wavelength step of $1 \mathrm{~nm}$ (each color corresponds to an image captured at a different wavelength step).

\section{CONCLUSION}

We have demonstrated a 16-element serpentine-based dispersive OPA at very near-IR wavelengths with $\mathrm{SiN}$ waveguide feeding. 2D beam steering is achieved by wavelength sweep, showing a tunability of $0.11 \% \mathrm{~nm}$ in the slow axis and $3.52^{\circ} \mathrm{nm}$ in the fast axis, with a beam divergence of $0.22^{\circ} \times 0.15^{\circ}$. The presented all-SiN serpentine-based dispersive OPA can handle high optical powers and has low phase errors and opens a full range of possibilities for optical beam steering, ranging and LiDAR in the very near-IR wavelengths regime.

\section{REFERENCES}

[1] Xavier Rottenberg et al., SPIE, Smart Photonic and Optoelectronic Integrated Circuits XXIII, vol. 11690, 2021

[2] Karel Van Accoleyen et al., IEEE Photon. Technol. Lett., vol. 23(17), 2011

[3] Christopher V. Poulton et al., OSA Opt. Letters, vol. 42(20), 2017

[4] Nathan Dostart et al., OSA Optica, vol.7(6), 2020

[5] Sarvagya Dwivedi et al., OSA Advanced Photonics Congress, paper IM4A.3, 2019

[6] Wim Bogaerts et al. IEEE J. Sel. Topics Quantum Electron., vol. 27(1), 2021

[7] Jeong Hwan Song et al., IEEE J. Light. Technol., vol. 38(12), 2020

[8] Abdul Rahim et al., IEEE J. Sel. Topics Quantum Electron., vol. 25(5), 2019 